\title{
Streamwise turbulence intensity formulation for flat-plate boundary layers
}

\author{
Ivan Marusic ${ }^{a)}$ and Gary J. Kunkel \\ Department of Aerospace Engineering and Mechanics, University of Minnesota, Minneapolis, \\ Minnesota 55455
}

(Received 11 February 2003; accepted 13 May 2003; published 2 July 2003)

\begin{abstract}
A similarity formulation is proposed to describe the streamwise turbulence intensity across the entire smooth-wall zero-pressure-gradient turbulent boundary layer. The formulation is an extension of the Marusic, Uddin, and Perry [Phys. Fluids 9, 3718 (1997)] formulation that was restricted to the outer region of the boundary layer, including the logarithmic region. The new formulation is found to agree very well with experimental data over a large range of Reynolds numbers varying from laboratory to atmospheric flows. The formulation is founded on physical arguments based on the attached eddy hypothesis, and suggests that the boundary layer changes significantly with Reynolds number, with an outer flow influence felt all the way down to the viscous sublayer. The formulation may also be used to explain why the empirical mixed scaling of DeGraaff and Eaton [J. Fluid Mech. 422, 319 (2000)] appears to work. (C) 2003 American Institute of Physics.
\end{abstract}

[DOI: $10.1063 / 1.1589014]$

Introduction. Over the past decade or so, increased controversy has arisen concerning the correct form of scaling for the streamwise turbulence intensity $\left(\overline{u^{2}}\right)$ in the near-wall region of the zero-pressure-gradient turbulent boundary layer. Studies such as Mochizuki and Nieuwstadt ${ }^{1}$ advocate that the classic "law of the wall" type formulation applies with inner variable scaling. That is, $\overline{u^{2}+}=f\left[z^{+}\right]$, where $u$ is the streamwise component of the fluctuating velocity and overbars denote long-time temporal averages. Here $\overline{u^{2}+}=\overline{u^{2}} / U_{\tau}^{2}$ and $z^{+}=z U_{\tau} / \nu$, where $U_{\tau}$ is the wall shear velocity, $z$ is the distance normal to the wall and $\nu$ is the kinematic viscosity of the fluid. Earlier surveys of data, such as $\mathrm{Coles}^{2}$ and Sreenivasan ${ }^{3}$ found that the classic formulation remains valid because any deviations could not be explained beyond the uncertainty in the measurements. More recent surveys by Gad-el-Hak and Bandyopadhyay ${ }^{4}$ and Fernholz and Finley ${ }^{5}$ indicated that Reynolds number effects are present, and therefore inner variable scaling alone is insufficient.

The major difficulty in drawing firm conclusions has been the accuracy of the measurements, which invariably are complicated by spatial resolution and other near-wall measurement issues. An excellent survey of these issues can be found in more recent papers by DeGraaff and Eaton ${ }^{6}$ and Metzger and Klewicki. ${ }^{7}$ Metzger and Klewicki considered data from laboratory and smooth wall atmospheric surface layer studies at the SLTEST facility on Utah's salt flats. They restricted their survey to datasets where $l^{+}<10$, where $l$ is the probe sensing length (hot-wire or LDA), and found a clear dependence on the Reynolds number. Most notably, the inner normalized rms streamwise velocity peak at $z^{+} \approx 15$ was found to increase with the Reynolds number approximately as

\footnotetext{
a)Telephone: (612) 625 3566; fax: (612) 626 1558; electronic mail: marusic@aem.umn.edu
}

$$
\max \left(u_{\mathrm{rms}}^{+}\right)=1.86+0.28 \log \left(R_{\theta}\right),
$$

where $R_{\theta}=\theta U_{1} / \nu$ is the Reynolds number based on momentum thickness and freestream velocity.

DeGraaff and Eaton ${ }^{6}$ conducted careful LDA measurements in a pressurized wind tunnel where $l^{+}<8$ for four Reynolds numbers and $l^{+}=17$ for their highest Reynolds number $R_{\theta}=31000$ or $\operatorname{Re}_{\tau}=13500$. Here $\operatorname{Re}_{\tau}=\delta_{c}^{+}=\delta_{c} U_{\tau} / \nu$ is the Karman number where $\delta_{c}$ is boundary layer thickness, obtained here by a curve-fit of the mean velocity profile to a law of the wall, law of the wake formulation (Ref. 8). From their experimental data DeGraaff and Eaton proposed a new near-wall mixed scaling where all near-wall data should collapse on a universal curve when plotted as $\overline{u^{2}} /\left(U_{1} U_{\tau}\right)$ versus $z^{+}$. Metzger et al. ${ }^{9}$ re-examined the data presented in Ref. 7 , including the atmospheric surface layer data, and concluded that mixed scaling does apply in the near-wall region for approximately $z^{+}<30$.

In this paper the similarity formulation of Marusic, Uddin, and Perry ${ }^{10}$ (referred to here on as MUP) is reconsidered. The MUP formulation was restricted to $z^{+}>100$ and therefore does not consider any of the near-wall viscous effects. It is based on the attached eddy hypothesis of Townsend ${ }^{11}$ and is given by

$$
\frac{\overline{u^{2}}}{U_{\tau}^{2}}=B_{1}-A_{1} \ln \left[\frac{z}{\delta_{c}}\right]-V_{g}\left[z^{+}, \frac{z}{\delta_{c}}\right]-W_{g}\left[\frac{z}{\delta_{c}}\right] .
$$

The formulation involves the asymptotic logarithmic law with a wake deviation $\left(W_{g}\right)$ in the outer region, and a viscous Reynolds number dependent deviation $\left(V_{g}\right)$ in the inner region. (The analytical expressions for $W_{g}$ and $V_{g}$ and values of the numerical constants are given in Ref. 10.) The formulation essentially provides a functional form for $\overline{u^{2}}$ as a universal function of $z^{+}$and $\operatorname{Re}_{\tau}\left(z / \delta_{c}=z^{+} / \operatorname{Re}_{\tau}\right)$. This is as expected since these are the only relevant dimensionless parameters for a flat plate boundary layer developing in a 
zero pressure gradient. In the following section an extension of the MUP formulation will be presented that applies across the entire boundary layer including the viscous near-wall region.

Extended formulation. The form of the new similarity formulation is derived here by considering physical arguments based on the attached eddy model as was used for the Marusic et al. ${ }^{10}$ formulation. The MUP formulation applies only in the logarithmic region and beyond where the inviscid attached eddies are considered. The attached eddy model (full details of which are given by Perry and Marusic ${ }^{12,13}$ ) is based on the attached eddy hypothesis of Townsend. ${ }^{11}$ The eddies are assumed to be geometrically similar with varying population density for different sizes of eddies. They are attached in the sense that their characteristic length is proportional to the distance the eddy extends above the wall. The height of the smallest attached eddy is assumed to scale with $\nu / U_{\tau}$, say $100 \nu / U_{\tau}$, while the largest attached eddies are assumed to be of size $\delta_{c}$, the boundary layer thickness. Therefore, it is immediately implied that an increase in Reynolds number, or Karman number $\left(\operatorname{Re}_{\tau}=\delta_{c} U_{\tau} / \nu\right)$, will result in a larger range of scales of attached eddies. This also explains why the MUP formulation shows an increase in $\overline{u^{2}}+$ at a fixed $z^{+}$in the $\log$ region. For example, at $z^{+}=150$ there will be more and more eddies above $z^{+}=150$ for higher values of $\mathrm{Re}_{\tau}$, and each of these attached eddies have some significant contribution to $\overline{u^{2}}+$ at $z^{+}=150$. This is not expected for the wall-normal turbulence intensity provided the attached eddies have a regular inclined shape extending above the viscous buffer zone. These trends were confirmed by Perry and Marusic ${ }^{12}$ using Biot-Savart calculations. These induced attached eddy motions are consistent with what Bradshaw ${ }^{14}$ and Townsend ${ }^{15}$ referred to as "inactive motions," although their argument that inactive motions carry no Reynolds shear stress need not apply.

The MUP formulation is extended to the near-wall viscous region by proposing that the Reynolds number dependent, outer-layer turbulence intensity acts as an effective forcing on the viscous buffer zone and sublayer, where the near-wall vortex formation processes are taking place. Therefore, for simplicity we consider essentially two components:

$$
\frac{\overline{u^{2}}}{U_{\tau}^{2}}=\left\{\begin{array}{l}
f_{1}\left[z^{+}\right] f_{T}\left[z^{+}, \operatorname{Re}_{\tau}\right], \quad \text { for } z^{+} \leqslant z_{\text {inner }}^{+} \\
f_{2}\left[z^{+}, \operatorname{Re}_{\tau}\right], \quad \text { for } z^{+} \geqslant z_{\text {outer }}^{+}
\end{array}\right.
$$

where the outer region part $f_{2}$ is the original MUP formulation as given by Eq. (1). The inner part of the layer is described by a universal function, $f_{1}\left[z^{+}\right]$, which is multiplied by a function $f_{T}\left[z^{+}, \operatorname{Re}_{\tau}\right]$ that accounts for the different levels of turbulence intensity forcing the near-wall viscous region. For simplicity, we complete the formulation by blending the two components between $z_{\text {inner }}^{+}<z^{+}<z_{\text {outer }}^{+}$with a gradient-matched cubic curve-fit. Here we tentatively take $z_{\text {inner }}^{+}=30$ and $z_{\text {outer }}^{+}=150$, the exact values of which are of secondary importance. Figure 1 shows a schematic of the new formulation. The MUP formulation $\left(f_{2}\right)$ is shown for several Reynolds numbers to highlight the increasing level of $\overline{u^{2}} / U_{\tau}^{2}$ above the near-wall viscous region. The inner region function $f_{1}$ shown on the figure corresponds to a reference

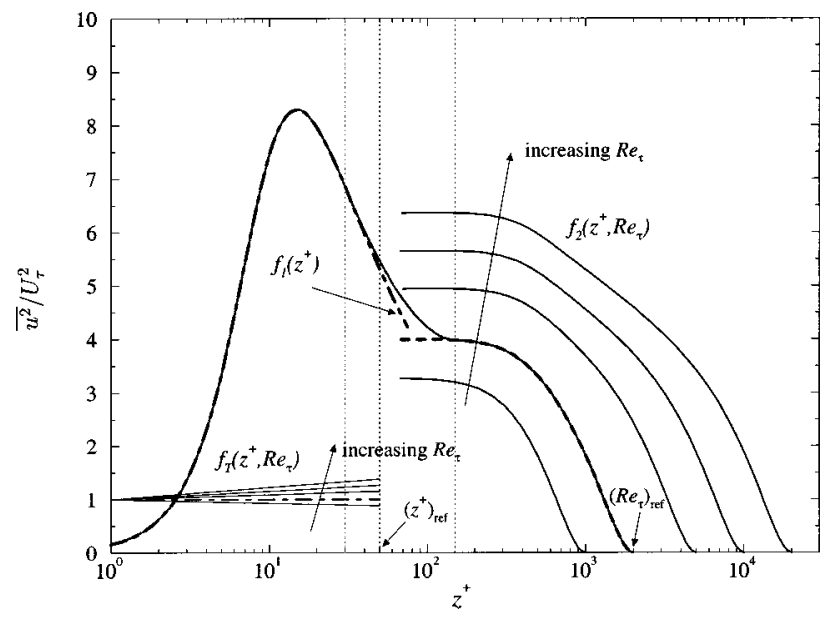

FIG. 1. Schematic of components in the new formulation.

Reynolds number for which $f_{T}=1$. Here, the nominal chosen value is $\left(\operatorname{Re}_{\tau}\right)_{\text {ref }}=2000$. An empirical curve fit for $f_{1}$ is obtained using one highly resolved experimental profile (Ref. 6 ). The functional form used to curve fit $f_{1}$ is

$$
f_{1}\left[z^{+}\right]=\frac{0.16\left(z^{+}\right)^{2}}{\left(1+a_{1}\left(z^{+}\right)^{2}\right)^{1 / 2}\left(1+\left(a_{2} z^{+}\right)^{2 a_{3}}\right)^{1 / 2}},
$$

where $a_{1}=0.008, a_{2}=0.115$, and $a_{3}=1.6$. The function was chosen so as to have the correct near-wall behavior, namely $f_{1} \rightarrow\left(0.4 z^{+}\right)^{2}$ as $z^{+} \rightarrow 0$. The scaling function $f_{T}\left[z^{+}, \operatorname{Re}_{\tau}\right]$ is also shown in the figure for the same Reynolds numbers as shown for $f_{2}$. It corresponds to a constant value at the edge of the viscous zone, labeled here as $\left(z^{+}\right)_{\text {ref }}$, which then simply drops off linearly in $\ln \left[z^{+}\right]$to a value of 1.0 at $z^{+}=1$. Here we choose $\left(z^{+}\right)_{\text {ref }}=50$. The functional form of $f_{T}$ for $z^{+} \geqslant 1$ is

$$
f_{T}\left[z^{+}, \operatorname{Re}_{\tau}\right]=1+(\alpha-1) \frac{\ln \left[z^{+}\right]}{\ln \left[\left(z^{+}\right)_{\mathrm{ref}}\right]},
$$

where $\alpha$ is the value of $f_{T}$ at $\left(z^{+}\right)_{\text {ref }}$, and it is obtained by taking the asymptotic form of $f_{2}$, that is

$$
\alpha=\frac{B_{1}-A_{1} \ln \left[\left(z^{+}\right)_{\mathrm{ref}} / \operatorname{Re}_{\tau}\right]}{B_{1}-A_{1} \ln \left[\left(z^{+}\right)_{\mathrm{ref}} /\left(\operatorname{Re}_{\tau}\right)_{\mathrm{ref}}\right]} .
$$

For $z^{+}<1$, the transition function is simply taken as $f_{T}=1$.

The resulting extended formulation is an analytical function which, while not very compact, is easy to code and process on a personal computer. Also, it should be noted that the functional forms of the curve-fits presented here are tentative and other curve-fit functions may work just as well. The important feature of the formulation is the functional relation given by Eq. (2).

Results and discussion. A comparison of formulation (2) with experimental data is shown in Fig. 2. The laboratory range Reynolds numbers correspond to the data of DeGraaff and Eaton, ${ }^{6}$ while the highest Reynolds numbers come from measurements on the Utah salt flats. The data from Metzger et $a l .{ }^{9}$ are as taken from Fig. 2 of their paper with $*$ indicating 10 independent, 5-minute runs and $\times$ the composite 50 minute averages. The conditions for these runs were re- 


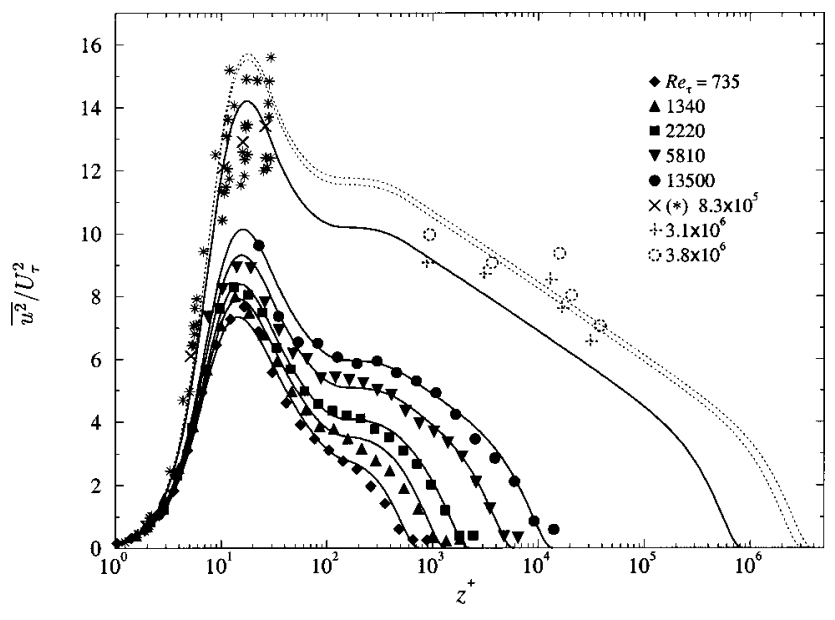

FIG. 2. A comparison of formulation (2) (lines) with experimental data. Solid symbols are from DeGraaff and Eaton (Ref. 6) and symbols * and $\times$ are from Metzger et al. (Ref. 9). Broken symbols are from authors' experimental study at the SLTEST atmospheric surface layer, with corresponding broken lines from Eq. (2).

garded as a nominally smooth wall (Refs. 7 and 9). Also included on the plot are data taken by the authors on the Utah salt flats. The data were acquired in August 2000 under neutrally stable, nominally rough wall conditions. Further experimental details concerning this study are given in Marusic and Kunkel. ${ }^{16}$ These data and the comparison with Eq. (2) are shown as broken lines since Eq. (2) does apply for both rough and smooth walls in the outer region (where $\left.\overline{u^{2}+}=f_{2}\left[z^{+}, \operatorname{Re}_{\tau}\right]\right)$ but only applies for smooth walls in the inner near-wall region.

In all cases the agreement between Eq. (2) and the experimental data in Fig. 2 is seen to be very good. A clear increase in the peak streamwise turbulence intensity is noted for increasing Reynolds numbers. Figure 3 shows the value of the peak $u_{\mathrm{rms}}^{+}=\left(\overline{u^{2}+}\right)^{1 / 2}$ as a function of Karman number $\operatorname{Re}_{\tau}$. Excellent agreement is seen with experimental data that are taken from the compilation of measurements for $l^{+}$

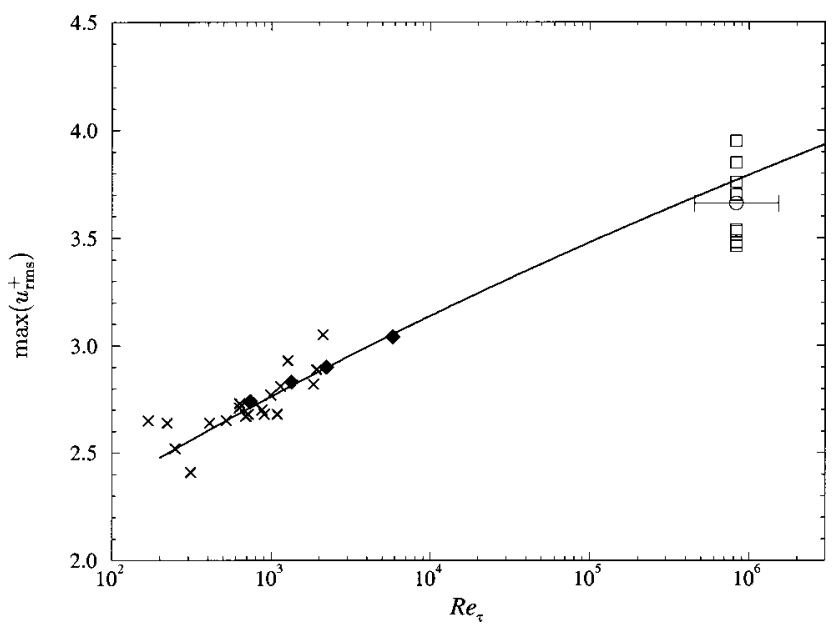

FIG. 3. Peak value of $u_{\mathrm{rms}}^{+}$as a function of Karman number. The solid line is from Eq. (2) and data points are as taken from Fig. 4 of Metzger and Klewicki (Ref. 7) where all measurements have sensing length $l^{+}<10$. The solid symbols are the data of DeGraaff and Eaton (Ref. 6).

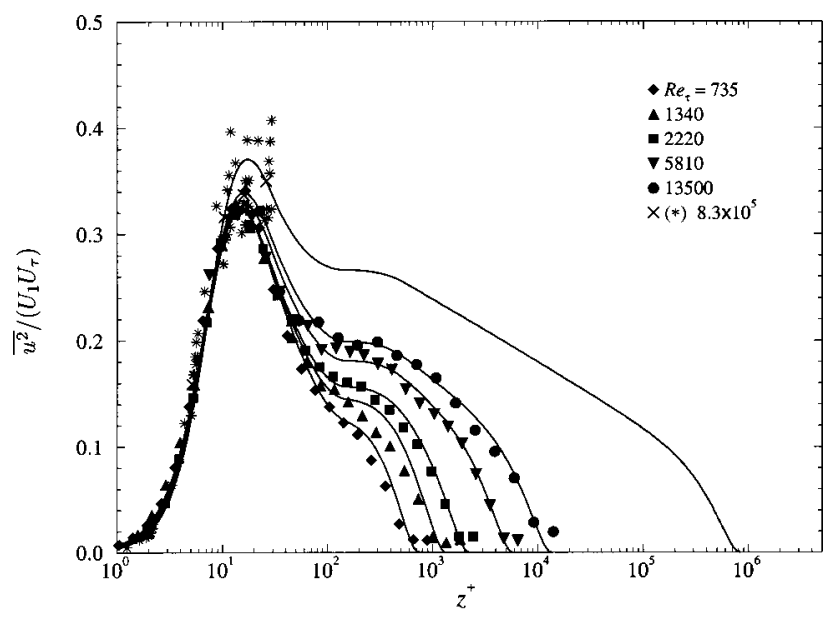

FIG. 4. Streamwise turbulence intensities normalized with mixed velocity scaling. Symbols and lines are as in Fig. 2.

$<10$ given in Refs. 7 and 9. This is in close agreement with what was empirically proposed by Metzger and Klewicki. ${ }^{7}$

The good agreement with experimental data also indicates support for the underlying physical basis of the formulation. That is, the inner-flow viscous region is directly influenced by outer-flow attached eddy motions, and this interaction changes significantly with Reynolds number. This relates directly to a discussion in the literature about the socalled inner/outer interaction in boundary layers. Our work lends support to the findings of Metzger and Klewicki ${ }^{7}$ and others that the outer region does have a direct effect on the near-wall region, all the way down to the viscous sublayer. This is in contrast with the view that viscous-region dynamics are "autonomous" and independent of the outer flow region. While the inner region can be self-sustaining in isolation, as shown from the numerical experiments of Jimenez and Pinelli ${ }^{17}$ and the dynamical modeling of Waleffe, ${ }^{18}$ it would seem that other mechanisms do play a significant role, especially at high Reynolds numbers.

An additional point concerning formulation (2) is that it may be used to explain why the empirical mixed scaling of DeGraaff and Eaton ${ }^{6}$ appears to work. Figure 4 shows the smooth wall data from Fig. 2 replotted with mixed coordinates. The experimental data collapse in the near-wall region within the scatter of the experimental uncertainty. The lines correspond to Eq. (2) for the different Reynolds numbers.

Strictly, the formulation will only give a true constant for peak $u_{\text {rms }}^{+}$at infinite $\operatorname{Re}_{\tau}$. This can be understood simply by considering a law of the wall and law of the wake formulation for the mean velocity. For example, using the formulation in Perry, Marusic and Jones, ${ }^{8}$

$$
\frac{U_{1}}{U_{\tau}}=\frac{1}{\kappa} \ln \left[\operatorname{Re}_{\tau}\right]+A-\frac{1}{3 \kappa}+\frac{2 \Pi}{\kappa},
$$

where $\kappa$ and $A$ are the logarithmic law of the wall constants and $\Pi$ is the Coles wake factor, which also becomes a constant at high $\operatorname{Re}_{\tau}$ for zero-pressure-gradient boundary layers. For high $\operatorname{Re}_{\tau}$ it is easy to show that from (2), 


$$
\left(\frac{\overline{u^{2}}}{U_{\tau}^{2}}\right)_{\max }=c_{0}+c_{1} \ln \left[\operatorname{Re}_{\tau}\right],
$$

where $c_{0}$ and $c_{1}$ are constants. Therefore, for high enough $\operatorname{Re}_{\tau}$ the logarithmic terms in (6) and (7) dominate and

$$
\left(\frac{\overline{u^{2}}}{U_{1} U_{\tau}}\right)_{\max } \rightarrow c_{1} \kappa .
$$

However, it should be noted that the Utah Reynolds numbers are already extremely high and that any practical application at such Reynolds numbers can no longer be regarded as hydrodynamically smooth.

Conclusion. A new streamwise turbulence intensity similarity formulation is proposed for the entire smooth-wall zero-pressure-gradient turbulent boundary layer. The formulation is based on the attached eddy hypothesis and the idea that the attached eddy motions in the log region and beyond impose a forcing on the viscous buffer zone and sublayer. This outer layer forcing yields the Reynolds number dependence in the inner portion of the layer. The new formulation is found to describe data well over a large range of Reynolds numbers varying from laboratory to atmospheric flows. The formulation also gives a theoretical basis for the empirical mixed scaling arguments of DeGraaff and Eaton. ${ }^{6}$

\section{ACKNOWLEDGMENTS}

The authors wish to acknowledge the support of the Packard Foundation and the National Science Foundation under Grants No. CTS-9983933 and No. ACI-9982274. The atmospheric measurements were made possible through the hospitality of Professor Joe Klewicki at the SLTEST site, which is funded by grants from the NSF and ONR, grant monitors M. Plesniak and R. Joslin, respectively.

${ }^{1}$ S. Mochizuki and F. T. M. Nieuwstadt, "Reynolds-number-dependence of the maximum in the streamwise velocity fluctuations in wall turbulence," Exp. Fluids 21, 218 (1996).
${ }^{2}$ D. Coles, "A model for flow in the viscous sublayer," in Proceedings of the Workshop on Coherent Structure of Turbulent Boundary Layers, edited by C. R. Smith and D. E. Abbott, Lehigh University, 1978.

${ }^{3}$ K. R. Sreenivasan, "The turbulent boundary layer," in Frontiers in Experimental Fluid Mechanics, edited by M. Gad el Hak (Springer-Verlag, Berlin, 1989).

${ }^{4}$ M. Gad-el-Hak and P. R. Bandyopadhyay, "Reynolds number effects in wall-bounded turbulent flows," Appl. Mech. Rev. 47, 307 (1994).

${ }^{5}$ H. H. Fernholz and P. J. Finley, "The incompressible zero-pressuregradient turbulent boundary layer: an assessment of the data," Prog. Aerosp. Sci. 32, 245 (1996).

${ }^{6}$ D. B. DeGraaff and J. K. Eaton, "Reynolds number scaling of the flat plate turbulent boundary layer," J. Fluid Mech. 422, 319 (2000).

${ }^{7}$ M. M. Metzger and J. C. Klewicki, "A comparative study of near-wall turbulence in high and low Reynolds number boundary layers," Phys. Fluids 13, 692 (2001).

${ }^{8}$ A. E. Perry, I. Marusic, and M. B. Jones, "On the streamwise evolution of turbulent boundary layers in arbitrary pressure gradients," J. Fluid Mech. 461, 61 (2002).

${ }^{9}$ M. M. Metzger, J. C. Klewicki, K. L. Bradshaw, and R. Sadr, "Scaling the near-wall axial turbulent stress in the zero pressure gradient boundary layer," Phys. Fluids 13, 1819 (2001).

${ }^{10}$ I. Marusic, A. K. M. Uddin, and A. E. Perry, "Similarity law for the streamwise turbulence intensity in zero-pressure-gradient turbulent boundary layers," Phys. Fluids 9, 3718 (1997).

${ }^{11}$ A. A. Townsend, The Structure of Turbulent Shear Flow (Cambridge University Press, Cambridge, 1976), Vol. 2.

${ }^{12}$ A. E. Perry and I. Marusic, "A wall-wake model for the turbulence structure of boundary layers. Part 1. Extension of the attached eddy hypothesis," J. Fluid Mech. 298, 361 (1995).

${ }^{13}$ I. Marusic and A. E. Perry, "A wall-wake model for the turbulence structure of boundary layers. Part 2. Further experimental support," J. Fluid Mech. 298, 389 (1995).

${ }^{14} \mathrm{P}$. Bradshaw, "The turbulence structure of equilibrium boundary layers," J. Fluid Mech. 29, 625 (1967).

${ }^{15}$ A. A. Townsend, "Equilibrium layers and wall turbulence," J. Fluid Mech. 11, 97 (1961).

${ }^{16}$ I. Marusic and G. J. Kunkel, "Turbulence intensity similarity laws for high Reynolds number boundary layers," in Reynolds Number Scaling in Turbulent Flow, edited by A. J. Smits (Kluwer Academic, Dordrecht, in press).

${ }^{17}$ J. Jimenez and A. Pinelli, "The autonomous cycle of near-wall turbulence," J. Fluid Mech. 389, 335 (1999).

${ }^{18} \mathrm{~F}$. Waleffe, "On a self-sustaining process in shear flows," Phys. Fluids $\mathbf{9}$, 883 (1997). 


\section{University Library}

\section{- M M N E R VA A gateway to Melbourne's research publications}

Minerva Access is the Institutional Repository of The University of Melbourne

Author/s:

Marusic, l;Kunkel, GJ

Title:

Streamwise turbulence intensity formulation for flat-plate boundary layers

Date:

2003-08-01

Citation:

Marusic, I. \& Kunkel, G. J. (2003). Streamwise turbulence intensity formulation for flat-plate boundary layers. PHYSICS OF FLUIDS, 15 (8), pp.2461-2464. https:// doi.org/10.1063/1.1589014.

Publication Status:

Published

Persistent Link:

http://hdl.handle.net/11343/34775 